\title{
Sitzung vom 12. April 1897.
}

Vorsitzender: Hr. C. Liebermann, Vice-Präsident.

Das Protocoll der letzten Sitzung wird genehmigt.

Der Vorsitzende theilt mit, dass die Deutsche chemische Gesellschaft seit ihrer letzten Sitzung einen schmerzlichen Verlust durch das Hinscheiden ihres Mitgliedes und Mitbegründers:

\section{Dr. Hug0 KunhFIM}

erlitten hat, welcher - erst $59 \mathrm{Jahr}$ alt - am 23. März d. J. allzufrüh seiner Familie, dem Freundeskreise und seiner ausgedehnten und hervorragenden Berufsthätigkeit entrissen worden ist.

Hugo Kunheim wurde als der einzige Sohn des bekannten chemischen Grossindustriellen Dr. Louis Kunheim am 17. Juni 1838 zu Berlin geboren. Nachdem er auf dem Friedrich-Wilhelms-Gymnasium hierselbst das Zeugniss der Reife erlangt hatte, studirte er Chemie in Berlin, Heidelberg und Göttingen, promovirte an letzterer Universität und trat dann, 1864 , in die damals schon in hoher Blüthe stehende Fabrik seines Vaters ein.

Diese Fabrik, eine der ältesten chemischen Fabriken Deutschlands und speciell Berlins, war von seinem Grossvater und Vater 1831 in kleinstem Umfange angelegt, 1834 aber durch Ankauf des Terrains am Kreuzberge bei Berlin erweitert worden, welches von da ab, ein halbes Jahrhundert lang, das sich immer mehr ausdehnende Kunheim'sche Etablissement aufnehmen sollte. Anfänglich wurden Beizen für die Textilindustrie dargestellt. $184+$ wurde die erste Schwefelsäurekammer errichtet und damit der Grundstein zu der bedeutsamen Stellung gelegt, welche die Fabrik bald darauf in der chemischen Grossindustrie Deutschlands einnabm and noch heute einnimmt.

Auch nach Hugo Kunheim's Eintritt in die Fabrik arbeitete dieselbe geräuschlos, aber unablässig an all' den grossen Veränderungen und Verbesserungen, welche die Industrie der Säuren und Alkalien im Laufe der Zeit durchgemacht hat. So führte u. a. Hugo Konheim den Deacon-Process in Deutschland ein, den er zur Gewinnung

Berichto d. D. chem. Geselischaft. Jahrg. $\mathbf{X X X}$. 
von chlorsaurem Kali benutzte. In der Zeit wuchs auch der äussere Umfang der Fabrik beträchtlich durch Anlegung der.Zweigfabriken in Niederschönweide und in Grube Ilse bei Senftenberg i. N.-L. sowie durch Ankauf des fiscalischen Alaunwerks in Freienwalde a/O., das in eine Ziegelei verwandelt wurde.

Hatte $\mathrm{Hu}$ go $\mathrm{K}$ unh eim schon lange als Theilhaber selbstständigen Antheil an der Leitung der Fabrik genommen, so fiel ihm bei dem 1878 erfolgten Tode seines Vaters die alleinige technische Leitung des grossen Unternehmens zu. Anfangs besorgte sein Freund Wartenberg noch den kaufmännischen Theil des Geschäfts. Später trennten sich die Freunde, und Kunbeim übernabm auch hierin die Fübrung.

Die gross angelegte Schöpfung, welche Hugo Kunbeim von seinem Vater überkommen hatte, wusste er nicht nur im Sinne desselben weiter auszubauen, sondern auch durch neu hinzueroberte Gebiete zu erweitern. Ebenso verstand er es aber auch, ganz oder örtlich unhaltbar Gewordenes fallen zu lassen, in dem klaren Bewusstsein, dass sich auch die Aufgaben einer Fabrik fortgesetzt ändern. So verschwand z. B. die Darstellung von Soda ganz aus dem Progranım der Fabrik; so wurde die sehr elegante Fabrication von reinem Schwefel, durch Extraction der Gasreinigungsmasse mit Schwefelkoblenstoff, wieder aufgegeben; die Gasreinigungsmasse dagegen theils zur Gewinnung von Schwefelsäure, teils zur Darstellung von Rhodanund Blutlaugensalz, Berlinerblau und den feiveren Sorten Pariserblau und Stahlblau in den Grossbetrieb aufgenommen. Später kam die technische Herstellung comprimirter Gase - Kohlensäure, Ammoniak, Chlor - an die Reihe. Flüssige Kohlensäure hat Kunheim, kurz nachdem sie Krupp in Essen zur Erzielung dichteren Gussstahls benutzt batte, zuerst (1882) in technischen Betrieb und in den Handel gebracht, indem er gleichzeitig auf deren vielseitige Anwendbarkeit in einer Broschüre hinwies. Die Aufnahme des Calciumcarbids und der Verarbeitung des Monazitsandes auf Thor- und Cer-Salze in seine Fabrication geben endlich daron Kunde, wie $\mathrm{Kunheim}$ bis in die letzte Zeit hinein alle chemischen Neuerungen nach der Richtung ihrer Verwerthbarkeit für den Grossbetrieb zu durchforschen bemüht war. Auch dem organischen Gebiet gegenüber verhielt er sich von jeher keineswegs ablehnend; schon früh gehörten die beiden Naphtole, Oxalsäure, Phtalsäure, später auch Citronensäure zu seinem Fabrikbetrieb.

Mehrfach verband sich auch $K u n h$ eim zur Lösung interessanter technisch-chemischer Probleme mit andern grossen Firmen, so namentlich zur Begründung einer elektrischen Grossindustrie durch Herstellung auf diesem Wege von Aluminium, Chlor und Pottasche, Calciumcarbid, welche Bestrebungen in der Gründung der Gesellschaft "Elektron* festere Gestalt annabmen. 
Eine hervorragende That Kunheim's, welche ibu eine Reihe von Jahren angestrengt beschäftigte, war auch die Ueberführung der älteren Fabrik am Kreuzberg, wo ihr die Grossstadt allmählich die beengendsten Fesseln anlegte, nach dem in der weiteren Umgebung Berlins gelegenen Nieder-Schönweide, welches durch seine gleicbzeitige Lage an der Spree und Eisenbahn für einen ausgedehnten Fabrikbetrieb besonders geeignet erschien. Wer je Gelegenheit gehabt hat, die schmucken Gebände uud sauberen Strassen dieser neuen, von der behaglichen Ruhe des Landlebens umgebenen, Fabrik mit den oft primitiven Bauten, den schlechten Wegen und der geräuschvollen Enge der alten Fabrik zu vergleichen, wird erkennen, wieviel klare Geistesarbeit und Schaffengfreudigkeit hier zum Zustandekommen einer allen technischen und hygienischen Ansprüchen der Neuzeit gemässen Anlage zusammengewirkt haben. Die Harmonie des Wohuhauses verschmilzt bier mit der rastlosen Thätigkeit der Arbeitsstätte; ein glückliches Spiegelbild vom Charakter des Erbauers.

Den grossen Aufschwung, welchen die Fabrik von Neuem unter Hugo Kunheim's Leitung genommen hat, zeigen auch die folgenden Daten ${ }^{1}$ ):

Wühreud 1878 die 4 Fabriken im Ganzen nur 400 Arbeiter beschäftigten, hat sich die Zahl der letzteren, trotz der inzwischen erfolgten Abzweigung der Ilse Bergbau-Gesellschaft, jetzt auf über 1000 vermehrt. 23 Dampfkessel, mit 2400 qm Heizfläche und einem Jahresconsum von $27000 \mathrm{t}$ Steinkohlen und $32000 \mathrm{t}$ Braunkohlen, treiben jetzt 35 Dampfmascbinen mit $950 \mathrm{H}$. P. und liefern den Fabriken den zum Kochen benöthigten Dampf. Zahlreiche Specialeisenbahnwagen für Gaswasser und Schwefelsäure, ferner 2 Dampfer und $14 \mathrm{Kähne} \mathrm{mit} \mathrm{mehr}$ als $1000 \mathrm{t}$ 'Tragfähigkeit dienen dem Waarentransport der Fabriken.

Vorstehendes beweist genugsam die grosse Arbeitslast, welche dem Verstorbenen oblag, zumal er an keiner Stelle die eigene Leitung aus der Hand gab. Rastlos beschäftigte ihn, bis in die Zeit seiner Erkrankung hinein, der Gedanke an zweckniässige Verbesserungen, Ergänzungen und Erweiterungen seines Fabrikbetriebes. Trotzdem fand er noch Zeit, sich seiner zahlreichen Familie, dem Freundeskreis und offentlichen Aemtern zu widmen. Lange war er italienischer Consul; er gehörte dem Aeltesten-Collegium der Berliner Kaufmannschaft, sowie dem Aufsichtsrath vieler bedeutender industrieller Unternehmungen an. Sein Verhältniss zu seinen Arbeitern und Angestellten war das denkbar beste, wie ein zahlreicher Stamm 25 und mehr Jabre

1) Für diese sowie für viele der vorstehenden Angaben ist der Verf. den langjährigen Betriebschemiker der Kunbeim'schen Fiabrik, Hrn. Dr. August L.ange, zu Dank verpflichtet. 
der Fabrik angehöriger Arbeiter und Beamten zeigt. Er war ihnen oft ein Berather, regte durch sein eigenes Beispiel Jeden zu freudiger und gesteigerter Arbeit an und verstand es auf die Individualităt des Einzelnen einzugehen.

Ein zablreiches Trauergefolge seiner Familie, Freunde, Untergebenen und Berufsgenossen gab dem Dahingeschiedenen das Geleit 20 seiner letzteu Rubestätte auf dem Friedrich-Werderschen Friedhof in der Bergmannstrasse, ganz nahe der Arbeitsstätte, an der er den grössten Theil seines thätigen Lebens gewirkt hat.

Die Versammlung ehrt das Andenken des Verstorbenen durch Erheben von den Sitzeu.

Der Vorsitzende begrüsst darauf die in der Sitzung anwesendes auswärtigen Mitglieder: Hrn. Dr. Hugo Müller aus London, Hrn. Prof. A. Bistrzycki aus Freiburg (Schweiz) und Hrn. Dr. Hauff aus Feuerbach.

Zu ausserordentlichen Mitgliedern werden verkündet die HHrn:

Friedrich, Fr., Nürnberg;

Vis, Dr. G. N., Freiburg i. B.;

Bosart, L. W., $\}$ Berlin;

Renwick, F. F., London;

Neumann, Dr. S., Budapest;

Piccinini, Dr. A., Bologna;

Lossen, F., Heidelberg;

Ponzio, Dr. G., Turin;

Bielecki, J., Manchester;

Kraft, Dr. B., Prag;

Dijken, Dr. B. van, Rotterdam;

Bänziger, Dr. E., St. Ludwig;

Wegeli, U.,

Heberlein, Ed., Zürich;

Liebknecht, O., Charlottenburg;

Koppel, J. van,

Lienau, H., iBerlin;

Wolf, E.,

Ry an, H., Galway;

Lehmkubl, Dr. J. N., Arnhem;

Samuel, E., München;

Brüll, J., Pardubitz;

Fireman, Dr. P., Washington;

Karlste in, A., Leipzig;

Berwerth, Prof. Dr. F., Wien; 


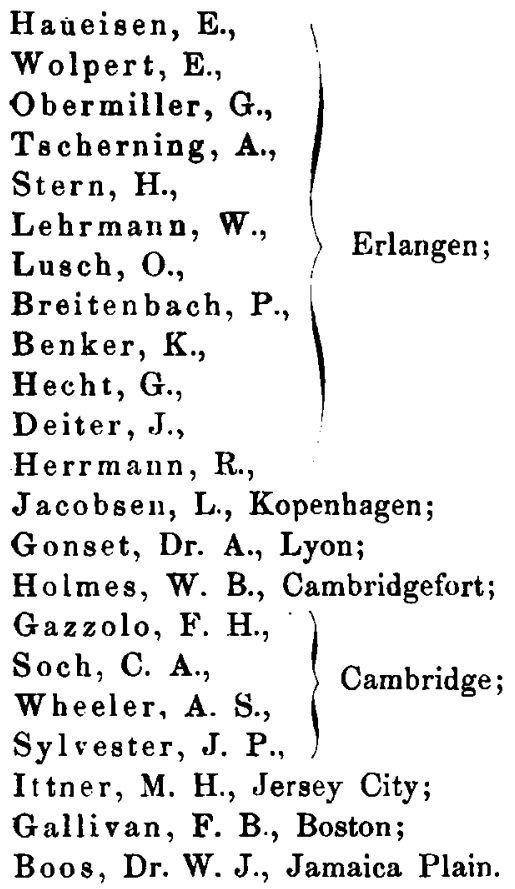

$\mathrm{Zu}$ ausserordentlichen Mitgliedern werden vorgeschlagen die HHra.:

Gad a mer, Dr. J., Privatdocent an der Universität, Marburg (durch E. Schmidt und O. Fritzsch);

How ard, Curtis C., Wilhelmstr. 39, III $\mathbf{~ B e r l i n ~ ( d u r c h ~ H . ~}$ Lichtenstein, Alfred F., Friedrich Wil- Wichelhans helmsstr. 7, pt. und E. Täuber);

Grosse, Dr. Siegfried P., W., Lützow-, Berlin (durch P. strasse 46

Wollny, Dr. R., NW., Werftstr. 8 R. Stelzner);

Trillich, Heinrich, Betriebsdirector, Uerdingen a. $\mathbf{R h}$. (durch C. Lintner und H. Kiliani).

Heymann. Stanislaw, Winkel i. Rheingal (durch F. Geromont and H. Goldenberg);

Hindermann, E., Rheingasse 66, Basel, (durch E. BamStiegelmann, Dr. A., Assistent am Poly- berger und technicum, Zürich

F. Feist);

Fürth, Dr. Otto Ritter von, Assistent am pbysiolog. Institut der Universität, Strassburg i/E. (durch F. Hofmeister und K. Spiro);

Meulen, Henri ter, Koornmarkt, Delft (durch S. Hoogewerff und L. Arnotein); 
Kimens, R. E., 6 Pienkna, Warschau (durch C. Lifbermann und H. Finkenbeiner);

Dieterich, Dr. Karl, Fabrikbenitzer. Helfenberg b. Dresden (durch R. Henriques uud P. Wolff);

Boltwood, Bertram B., Sheffield Laboratory, New Haven, Walden, Dr. Percy, P.,
Barnes, Bayard, Conn., U.S.A. (durch H. L. Metcalf, H. F., Wells and H. L. Wheeler);

Betts, Anson G., Lansingburgh, New York (durch H. L. Wells und H. L. Wheeler);

Dietz, Dr. Rudolf, NW., Flemmingstr. 12, Berlin (durch W. Will und F. Mylius);

Combes, Prof. Charles, 15 Rue Bara, Paris (durch P. A. Guye and C. Graebe);

Liebermann, Hans, W., Bellevuestr. 8, Berlin (durch C. Liebermann und A. Pinner).

Der stellvertretende Schrifttührer bemerkt, dass in Folge der Veröffentlichung ') des an den Präsidenten der Gesellscbaft gerichteten Dankschreibens des Hrn. C. Scheibler mehrere Mitglieder den Wunsch ansgesprochen baben, Näheres darüber zu erfahren, in welcher Weise Hr. C. Sicheibler - der Mitbegründer und stete Förderer unserer Gesellschaft - vom Vorstande zu seinem 70. Geburtstage begrüsst worden ist. Es sei daher mitgetheilt, dass sich am 16. Februar eine Deputation des Vorstandes - bestehend aus den HHrn. E. Fischer, C. Liebermann, A. Pinner, F. Tiemano und H. Wichelhaus -, welcher sich eine grössere Zabl anderer Vorstandsmitglieder angeschlossen hatte, in die Wohnung des Gefeierten begab. Nach einer Ansprache des stellvertretenden Vorsitzenden, Hin. E. Fischer, wurde eine kalligraphisch ausgeführte und künstlerisch ausgeschmückte Adresse überreicbt, welche $\mathbf{H r}$. Wichelbaus in Auftrage des Vorstandes verfasst hatte und nunmehr auch vortrug.

Die Adresse hat folgrnden Wortlaut:

2An diesem festlichen Tage begrüsst Sie die Deutsche chemische Gesellschaft durch ihren Vorstand als Einen ibrer Begründer, als ibr altbewährtes Vorstandsmitglied, als Einen der ersten, welche wissenschaftliche Arbeiten in ihren Berichten veröffentlichten.

Sie blicken beute auf ein arbeitsames und erfolgreiches Leben zarück. Mehr als 100 Abhandlungen zeugen von Ibrer geistigen

3) Diese Beriohte 30, 463. - Daselbst lies Z. 6 ron oben; 16. Februar statt 15. Fobruar. 
Thätigkeit, und wenn die Forscher genannt werden, welche sich durch Untersuchung der Kohlenhydrate Verdienst erworben, welche neue Zuckerarten gefunden und beschrieben haben, so ist der Name

\section{Carl Scheibler}

einer der ersten.

Sie haben die Saccharose in den Rüben za bestimmen gelehrt, wie es vorher nicht mbglich war, sie durch alle $\mathbf{A b}$ schuitte des Darstellungsverfahrens verfolgt und gezeigt, wie das Verbältniss von Rohzucker und Raffinade ermittelt wird, Sie haben nicht geduldet, dass sie als minderwerthig erscheinender Rückstand vernachlässigt werd : Sacharate aus Melasse hergestellt und nutzbar gemacht * haben, ist einer Ihrer schönsten Erfolge.

Wie Marggraf"s Beobachtungen und Achard's unermüdlicher Eifer die Gewinnung des Zuckers aus den Früchten des heimischen Bodens ermöglichten, so haten Ihre Arbeiten dazu beigetragen, diesem Betriebe das Gepräge einer hochstehenden, alle Hülfsmittel der Chemie und Physik benutzenden Industrie zu geben. Jahrzehnte hindurch haben Sie auf diesem Gebiete als Leiter eines Laboratoriums und Herausgeber von Zeitschriften einen segensreichen Einfluss ausgeübt. Sie haben Schüler herangebildet, welche durch sachkundige Hülfe allen Aufgaben der ZuckerIndustrie gerecht wurden.

Dabei hat sich Ihr forschender Blick niemals auf das Gebiet Ihrer besonderen Thätigkeit beschränkt. Ihre Entdeckung des Betaīns hăngt mit früheren Untersuchungen über Phosphorwolframsäure zusammen, die Aufklärung der Natur der Honigsteinsäure haben Sie angeregt und die Kenntniss der neueren Sprengstoffe erweitert.

Wir aber, die wir seit vielen Jahren mit Ihnen über Angelegenheiten, nicht nur wissenschaftlicher, sondern auch persönlicher Art berathen, keunen und schätzen noch andere Seiten Ihres Wesens.

„,Humani nihil a me alienum puto“.

können Sie sagen.

Was nur der menschliche Geist ersinnt nnd schafft, findet bei Ihnen Verständniss und Würdigung. Die Verdienste Anderer wissen Sie zu schätzen und Freundschaft zu halten.

So ist denn Ihr Name eng verknüpft mit der Entstehung der Denkmäler Liebig's und den Bestrebungen, ein HofmannHaus zu erricbten, so ist es der Dank für vielseitige Thätigkeit in 
unserem Kreise, der sich mit der Anerkennung Ibrer wissenschaftlichen Arbeiten und mit dem Wunsche verbindet, dass Ihre Wirksamkeit uns und der Wissenschaft unverändert erhalten bleiben möge."

Berlin, den 16. Februar 1897.

\section{Der Vorstand der Deutschen Chemischen Gesellschaft.}
Ferd. Tiemanu
Schriftführer.
Victor Meyer
Präsident.
Adolf Pinner
Schriftführer.

Für die Bibliothek sind als Geschenke eingegangen:

26. v. Fehling, H. Neues Handwörterbuch der Chemie. Fortges. von O. Hell. J.frg. 81. Braunscliweig 1897 .

858. Jannasch, Paul. Practischer Leitfaden der Gewichtsaualyse. Leipzig. 1897.

859. Classen, Alexander. Qnantitative Analyse durch Elektrolyse. 4. Aufl. Berlin 1897.

860. Jean, Ferd. \& G. Nercier. Reagention für specielle chemische and pharmaceutische Zwecke. Uebersetzt von P. Duden. Weimar 1897.

861. Fischer, Ferd. Das Studium der technischen Clemie an den Universitäten und technischen Hochschulen Deutschlands und das ChemikerExamen. Braunschweig 1897.

862. Goldschmidt, F., Chr. Heinzerling, Helbig, E. Roth, Th. Weyl: Hygiene der chemischen Grossindustrie. Jena 1896.

773. Sammlung elemischer und chemisch-technischer Vorträge. Hrsggbn. von F. B. Ahrens. 1l. Bd., left 2: Der künstliche Aufliau der Alkaloide von Max Scholtz. Stuttgart 1897.

703. Beilstein, F. Handluch der organischen Chemie. 3. Aufl. Lfrg. 73-74. Hamburg und Leipzig 1897.

Der Vorsitzende:

C. Liebermann.
Der Schriftführer:
A. Pinner. 